\title{
Spectral asymptotics of Euclidean quantum gravity with diff-invariant boundary conditions
}

\author{
Giampiero Esposito ${ }^{1,2}$ Guglielmo Fucci ${ }^{2}$ Alexander Yu. Kamenshchik ${ }^{3,4}$ Klaus Kirsten ${ }^{5}$ \\ ${ }^{1}$ Istituto Nazionale di Fisica Nucleare, Sezione di Napoli, \\ Complesso Universitario di Monte S. Angelo, \\ Via Cintia, Edificio N', 80126 Napoli, Italy \\ ${ }^{2}$ Dipartimento di Scienze Fisiche, Complesso Universitario di Monte S. Angelo, \\ Via Cintia, Edificio N', 80126 Napoli, Italy \\ ${ }^{3}$ Dipartimento di Scienze Fisiche e Matematiche, \\ Università dell'Insubria, Como, Italy \\ ${ }^{4}$ L.D. Landau Institute for Theoretical Physics of \\ the Russian Academy of Sciences, Moscow, Russia \\ ${ }^{5}$ Department of Mathematics, Baylor University, Waco TX 76798, USA
}




\begin{abstract}
A general method is known to exist for studying Abelian and non-Abelian gauge theories, as well as Euclidean quantum gravity, at one-loop level on manifolds with boundary. In the latter case, boundary conditions on metric perturbations $h$ can be chosen to be completely invariant under infinitesimal diffeomorphisms, to preserve the invariance group of the theory and BRST symmetry. In the de Donder gauge, however, the resulting boundary-value problem for the Laplace type operator acting on $h$ is known to be self-adjoint but not strongly elliptic. The latter is a technical condition ensuring that a unique smooth solution of the boundary-value problem exists, which implies, in turn, that the global heat-kernel asymptotics yielding one-loop divergences and oneloop effective action actually exists. The present paper shows that, on the Euclidean four-ball, only the scalar part of perturbative modes for quantum gravity are affected by the lack of strong ellipticity. Further evidence for lack of strong ellipticity, from an analytic point of view, is therefore obtained. Interestingly, three sectors of the scalar-perturbation problem remain elliptic, while lack of strong ellipticity is "confined" to the remaining fourth sector. The integral representation of the resulting $\zeta$-function asymptotics is also obtained; this remains regular at the origin by virtue of a spectral identity here obtained for the first time.
\end{abstract}

PACS: 03.70.+k, 04.60.Ds 


\section{INTRODUCTION}

The study of gauge theories and quantum gravity on manifolds with boundary is motivated by the problems of quantum cosmology [1] and quantum field theory under the influence of external conditions [2], and several efforts have been produced in the literature to study boundary conditions and one-loop semiclassical properties within this framework 3]. In our paper we are interested in boundary conditions for metric perturbations that are completely invariant under infinitesimal diffeomorphisms, since they are part of the general scheme according to which the boundary conditions are preserved under the action of the symmetry group of the theory [4, 5, 6]. In field-theoretical language, this means setting to zero at the boundary that part $\pi A$ of the gauge field $A$ that lives on the boundary $\mathcal{B}(\pi$ being a projection operator):

$$
[\pi A]_{\mathcal{B}}=0
$$

as well as the gauge-fixing functional,

$$
[\Phi(A)]_{\mathcal{B}}=0
$$

and the whole ghost field

$$
[\varphi]_{\mathcal{B}}=0
$$

For Euclidean quantum gravity, Eq. (1.1) reads as

$$
\left[h_{i j}\right]_{\mathcal{B}}=0
$$

where $h_{i j}$ are perturbations of the induced three-metric. To arrive at the gravitational counterpart of Eqs. (1.2) and (1.3), note first that, under infinitesimal diffeomorphisms, metric perturbations $h_{\mu \nu}$ transform according to

$$
\widehat{h}_{\mu \nu} \equiv h_{\mu \nu}+\nabla_{(\mu} \varphi_{\nu)}
$$

where $\nabla$ is the Levi-Civita connection on the background four-geometry with metric $g$, and $\varphi_{\nu} d x^{\nu}$ is the ghost one-form (strictly, our presentation is simplified: there are two independent ghost fields obeying Fermi statistics, and we will eventually multiply by -2 the effect of $\varphi_{\nu}$ to take this into account). In geometric language, the infinitesimal variation

$\delta h_{\mu \nu} \equiv \widehat{h}_{\mu \nu}-h_{\mu \nu}$ is given by the Lie derivative along $\varphi$ of the four-metric $g$. For manifolds with boundary, Eq. (1.5) implies that [7, 8]

$$
\widehat{h}_{i j}=h_{i j}+\varphi_{(i \mid j)}+K_{i j} \varphi_{0},
$$


where the stroke denotes three-dimensional covariant differentiation tangentially with respect to the intrinsic Levi-Civita connection of the boundary, while $K_{i j}$ is the extrinsiccurvature tensor of the boundary. Of course, $\varphi_{0}$ and $\varphi_{i}$ are the normal and tangential components of the ghost, respectively. By virtue of Eq. (1.6), the boundary conditions (1.4) are "gauge invariant", i.e.

$$
\left[\widehat{h}_{i j}\right]_{\mathcal{B}}=0
$$

if and only if the whole ghost field obeys homogeneous Dirichlet conditions, so that

$$
\begin{aligned}
& {\left[\varphi_{0}\right]_{\mathcal{B}}=0,} \\
& {\left[\varphi_{i}\right]_{\mathcal{B}}=0 .}
\end{aligned}
$$

The conditions (1.8) and (1.9) are necessary and sufficient since $\varphi_{0}$ and $\varphi_{i}$ are independent, and three-dimensional covariant differentiation commutes with the operation of restriction to the boundary. We are indeed assuming that the boundary $\mathcal{B}$ is smooth and not totally geodesic, i.e. $K_{i j} \neq 0$. However, for totally geodesic manifolds, having $K_{i j}=0$, the condition (1.8) is no longer necessary.

On imposing boundary conditions on the remaining set of metric perturbations, the key point is to make sure that the invariance of such boundary conditions under the infinitesimal transformations (1.5) is again guaranteed by (1.8) and (1.9), since otherwise one would obtain incompatible sets of boundary conditions on the ghost field. Indeed, on using the DeWittFaddeev-Popov formalism for the $\langle$ out $|$ in $\rangle$ amplitudes of quantum gravity, it is necessary to use a gauge-averaging term in the Euclidean action, of the form [9]

$$
I_{g . a .}=\frac{1}{16 \pi G} \int_{\mathcal{M}} \frac{\Phi_{\nu} \Phi^{\nu}}{2 \alpha} \sqrt{\operatorname{det} g} d^{4} x
$$

where $\Phi_{\nu}$ is any functional which leads to self-adjoint (elliptic) operators on metric and ghost perturbations. One then finds that

$$
\delta \Phi_{\mu}(h) \equiv \Phi_{\mu}(h)-\Phi_{\mu}(\widehat{h})=\mathcal{F}_{\mu}^{\nu} \varphi_{\nu}
$$

where $\mathcal{F}_{\mu}^{\nu}$ is an elliptic operator that acts linearly on the ghost field. Thus, if one imposes the boundary conditions

$$
\left[\Phi_{\mu}(h)\right]_{\mathcal{B}}=0
$$


and if one assumes that the ghost field can be expanded in a complete orthonormal set of eigenfunctions $u_{\nu}^{(\lambda)}$ of $\mathcal{F}_{\mu}^{\nu}$ which vanish at the boundary, i.e.

$$
\begin{gathered}
\mathcal{F}_{\mu}{ }^{\nu} u_{\nu}^{(\lambda)}=\lambda u_{\mu}^{(\lambda)}, \\
\varphi_{\nu}=\sum_{\lambda} C_{\lambda} u_{\nu}^{(\lambda)}, \\
{\left[u_{\mu}^{(\lambda)}\right]_{\mathcal{B}}=0,}
\end{gathered}
$$

the boundary conditions (1.12) are automatically gauge-invariant under the Dirichlet conditions (1.8) and (1.9) on the ghost.

Having obtained the general recipe expressed by Eqs. (1.4) and (1.12), we can recall what they imply on the Euclidean four-ball. This background is relevant for one-loop quantum cosmology in the limit of small three-geometry on the one hand [10], and for spectral geometry and spectral asymptotics on the other hand [11]. As shown in Ref. [7], if one chooses the de Donder gauge-fixing functional

$$
\Phi_{\mu}(h)=\nabla^{\nu}\left(h_{\mu \nu}-\frac{1}{2} g_{\mu \nu} g^{\rho \sigma} h_{\rho \sigma}\right),
$$

which has the virtue of leading to an operator of Laplace type on $h_{\mu \nu}$ in the one-loop functional integral, Eq. (1.12) yields the mixed boundary conditions

$$
\begin{gathered}
{\left[\frac{\partial h_{00}}{\partial \tau}+\frac{6}{\tau} h_{00}-\frac{\partial}{\partial \tau}\left(g^{i j} h_{i j}\right)+\frac{2}{\tau^{2}} h_{0 i}^{\mid i}\right]_{\mathcal{B}}=0,} \\
{\left[\frac{\partial h_{0 i}}{\partial \tau}+\frac{3}{\tau} h_{0 i}-\frac{1}{2} \frac{\partial h_{00}}{\partial x^{i}}\right]_{\mathcal{B}}=0 .}
\end{gathered}
$$

In Refs. [3, 7], the boundary conditions (1.4), (1.17) and (1.18) were used to evaluate the full one-loop divergence of quantized general relativity on the Euclidean four-ball, including all $h_{\mu \nu}$ and all ghost modes. However, the meaning of such a calculation became unclear after the discovery in Ref. [6] that the boundary-value problem for the Laplacian $P$ acting on metric perturbations is not strongly elliptic by virtue of tangential derivatives in the boundary conditions (1.17) and (1.18). Strong ellipticity 11] is a technical requirement ensuring that a unique smooth solution of the boundary-value problem exists which vanishes at infinite geodesic distance from the boundary (see Appendix A). If it is fulfilled, this ensures 
that the $L^{2}$ trace of the heat semigroup $e^{-t P}$ exists, with the associated global heat-kernel asymptotics that yields one-loop divergence and one-loop effective action. However, when strong ellipticity does not hold, the $L^{2}$ trace of $e^{-t P}$ acquires a singular part [6], and hence it is unclear how to attach a meaning to $\zeta$-function calculations.

All of this has motivated our analysis, which therefore starts in Sec. II with the modeby-mode form of the boundary conditions (1.4), (1.8), (1.9), (1.17) and (1.18) with the resulting eigenvalue conditions. Section III studies the matrix for coupled scalar modes, while Sec. IV obtains the first pair of resulting scalar-mode $\zeta$-functions and Sec. V studies the remaining elliptic and non-elliptic parts of spectral asymptotics. Results and open problems are described in Sec. VI, while technical details are given in the Appendices.

\section{EIGENVALUE CONDITIONS ON THE FOUR-BALL}

On the Euclidean four-ball, which can be viewed as the portion of flat Euclidean fourspace bounded by a three-sphere of radius $q$, metric perturbations $h_{\mu \nu}$ can be expanded in terms of hyperspherical harmonics as [12, 13]

$$
\begin{gathered}
h_{00}(x, \tau)=\sum_{n=1}^{\infty} a_{n}(\tau) Q^{(n)}(x), \\
h_{0 i}(x, \tau)=\sum_{n=2}^{\infty}\left[b_{n}(\tau) \frac{Q_{\mid i}^{(n)}(x)}{\left(n^{2}-1\right)}+c_{n}(\tau) S_{i}^{(n)}(x)\right], \\
h_{i j}(x, \tau)=\sum_{n=3}^{\infty} d_{n}(\tau)\left[\frac{Q_{\mid i j}^{(n)}(x)}{\left(n^{2}-1\right)}+\frac{c_{i j}}{3} Q^{(n)}(x)\right]+\sum_{n=1}^{\infty} \frac{e_{n}(\tau)}{3} c_{i j} Q^{(n)}(x) \\
+\sum_{n=3}^{\infty}\left[f_{n}(\tau)\left(S_{i \mid j}^{(n)}(x)+S_{j \mid i}^{(n)}(x)\right)+k_{n}(\tau) G_{i j}^{(n)}(x)\right],
\end{gathered}
$$

where $\tau \in[0, q]$ and $Q^{(n)}(x), S_{i}^{(n)}(x)$ and $G_{i j}^{(n)}(x)$ are scalar, transverse vector and transversetraceless tensor hyperspherical harmonics, respectively, on a unit three-sphere with metric $c_{i j}$. By insertion of the expansions (2.1)-(2.3) into the eigenvalue equation for the Laplacian acting on $h_{\mu \nu}$, and by setting $\sqrt{E} \rightarrow i M$, which corresponds to a rotation of contour in the $\zeta$-function analysis [14], one finds the modes as linear combinations of modified Bessel functions of first kind according to 13 .

$$
a_{n}(\tau)=\frac{1}{\tau}\left[\gamma_{1} I_{n}(M \tau)+\gamma_{3} I_{n-2}(M \tau)+\gamma_{4} I_{n+2}(M \tau)\right],
$$




$$
\begin{gathered}
b_{n}(\tau)=\gamma_{2} I_{n}(M \tau)+(n+1) \gamma_{3} I_{n-2}(M \tau)-(n-1) \gamma_{4} I_{n+2}(M \tau), \\
c_{n}(\tau)=\varepsilon_{1} I_{n+1}(M \tau)+\varepsilon_{2} I_{n-1}(M \tau), \\
d_{n}(\tau)=\tau\left[-\gamma_{2} I_{n}(M \tau)+\frac{(n+1)}{(n-2)} \gamma_{3} I_{n-2}(M \tau)+\frac{(n-1)}{(n+2)} \gamma_{4} I_{n+2}(M \tau)\right], \\
e_{n}(\tau)=\tau\left[\left(3 \gamma_{1}-2 \gamma_{2}\right) I_{n}(M \tau)-\gamma_{3} I_{n-2}(M \tau)-\gamma_{4} I_{n+2}(M \tau)\right] \\
f_{n}(\tau)=\tau\left[-\frac{\varepsilon_{1}}{(n+2)} I_{n+1}(M \tau)+\frac{\varepsilon_{2}}{(n-2)} I_{n-1}(M \tau)\right] \\
k_{n}(\tau)=\alpha_{1} \tau I_{n}(M \tau) .
\end{gathered}
$$

Modified Bessel functions of second kind are not included to ensure regularity at the origin $\tau=0$. Moreover, normal and tangential components of the ghost field admit the following expansion on the four-ball:

$$
\begin{gathered}
\varphi_{0}(x, \tau)=\sum_{n=1}^{\infty} l_{n}(\tau) Q^{(n)}(x), \\
\varphi_{i}(x, \tau)=\sum_{n=2}^{\infty}\left[m_{n}(\tau) \frac{Q_{\mid i}^{(n)}(x)}{\left(n^{2}-1\right)}+p_{n}(\tau) S_{i}^{(n)}(x)\right],
\end{gathered}
$$

where the ghost modes $l_{n}(\tau), m_{n}(\tau)$ and $p_{n}(\tau)$ are found to read as [13]

$$
\begin{gathered}
l_{n}(\tau)=\frac{1}{\tau}\left[\kappa_{1} I_{n+1}(M \tau)+\kappa_{2} I_{n-1}(M \tau)\right], \\
m_{n}(\tau)=-(n-1) \kappa_{1} I_{n+1}(M \tau)+(n+1) \kappa_{2} I_{n-1}(M \tau), \\
p_{n}(\tau)=\theta I_{n}(M \tau) .
\end{gathered}
$$

At this stage, the boundary conditions (1.4), (1.17), (1.18), (1.8) and (1.9) can be reexpressed in terms of metric and ghost modes as

$$
\frac{d a_{n}}{d \tau}+\frac{6}{\tau} a_{n}-\frac{1}{\tau^{2}} \frac{d e_{n}}{d \tau}-\frac{2}{\tau^{2}} b_{n}=0 \text { on } S^{3},
$$




$$
\begin{gathered}
\frac{d b_{n}}{d \tau}+\frac{3}{\tau} b_{n}-\frac{\left(n^{2}-1\right)}{2} a_{n}=0 \text { on } S^{3}, \\
\frac{d c_{n}}{d \tau}+\frac{3}{\tau} c_{n}=0 \text { on } S^{3}, \\
d_{n}=e_{n}=f_{n}=k_{n}=l_{n}=m_{n}=p_{n}=0 \text { on } S^{3} .
\end{gathered}
$$

Furthermore, the formulae (2.4)-(2.10) and (2.13)-(2.15) can be used to obtain homogeneous linear systems that yield, implicitly, the eigenvalues of our problem. The conditions for finding non-trivial solutions of such linear systems are given by the vanishing of the associated determinants; these yield the eigenvalue conditions $\delta(E)=0$, i.e. the equations obeyed by the eigenvalues by virtue of the boundary conditions. For the purpose of a rigorous analysis, we need the full expression of such eigenvalue conditions for each set of coupled modes. Upon setting $\sqrt{E} \rightarrow i M$, we denote by $D(M q)$ the counterpart of $\delta(E)$, bearing in mind that, strictly, only $\delta(E)$ yields implicitly the eigenvalues, while $D(M q)$ is more convenient for $\zeta$-function calculations [14].

To begin, the decoupled vector mode $c_{2}(\tau)=I_{3}(M \tau)$ obeys the Robin boundary condition (2.18), which yields

$$
D(M q)=I_{2}(M q)+I_{4}(M q)+\frac{6}{M q} I_{3}(M q)
$$

with degeneracy 6. Coupled vector modes $c_{n}(\tau)$ and $f_{n}(\tau)$ obey the boundary conditions (2.18) and (2.19), and hence the corresponding $D(M q)$ reads as

$$
\begin{aligned}
D_{n}(M q) & =I_{n-1}(M q)\left(I_{n}(M q)+I_{n+2}(M q)+\frac{6}{M q} I_{n+1}(M q)\right) \\
& +\frac{(n-2)}{(n+2)} I_{n+1}(M q)\left(I_{n-2}(M q)+I_{n}(M q)+\frac{6}{M q} I_{n-1}(M q)\right),
\end{aligned}
$$

with degeneracy $2\left(n^{2}-1\right)$, for all $n \geq 3$.

The scalar modes

$$
\begin{aligned}
& a_{1}(\tau)=\frac{1}{\tau}\left(\gamma_{1} I_{1}(M \tau)+\gamma_{4} I_{3}(M \tau)\right), \\
& e_{1}(\tau)=\tau\left(3 \gamma_{1} I_{1}(M \tau)-\gamma_{4} I_{3}(M \tau)\right),
\end{aligned}
$$


obey the boundary conditions

$$
\begin{gathered}
\frac{d a_{1}}{d \tau}+\frac{6}{\tau}-\frac{1}{\tau^{2}} \frac{d e_{1}}{d \tau}=0 \text { at } \tau=q \\
e_{1}(q)=0
\end{gathered}
$$

which imply

$$
\begin{aligned}
D(M q) & =20 I_{1}(M q) I_{3}(M q)-M q\left(I_{0}(M q)+I_{2}(M q)\right) I_{3}(M q) \\
& +3 M q I_{1}(M q)\left(I_{2}(M q)+I_{4}(M q)\right)
\end{aligned}
$$

with degeneracy 1.

The scalar modes

$$
\begin{gathered}
a_{2}(\tau)=\frac{1}{\tau}\left[\gamma_{1} I_{2}(M \tau)+\gamma_{4} I_{4}(M \tau)\right], \\
b_{2}(\tau)=\gamma_{2} I_{2}(M \tau)-\gamma_{4} I_{4}(M \tau), \\
e_{2}(\tau)=\tau\left[\left(3 \gamma_{1}-2 \gamma_{2}\right) I_{2}(M \tau)-\gamma_{4} I_{4}(M \tau)\right],
\end{gathered}
$$

obey the boundary conditions (2.16), (2.17) and (2.19) with $n=2$, and hence yield the determinant

$$
D(M q)=\operatorname{det}\left(\begin{array}{lll}
I_{2}(M q)-M q I_{2}^{\prime}(M q) & M q I_{2}^{\prime}(M q) & 4 I_{4}(M q)+M q I_{4}^{\prime}(M q) \\
3 I_{2}(M q) & -\left(2 M q I_{2}^{\prime}(M q)+6 I_{2}(M q)\right) & 2 M q I_{4}^{\prime}(M q)+9 I_{4}(M q) \\
3 I_{2}(M q) & -2 I_{2}(M q) & -I_{4}(M q)
\end{array}\right),
$$

with degeneracy 4.

For all $n \geq 3$, coupled scalar modes $a_{n}, b_{n}, d_{n}, e_{n}$ obey the boundary conditions (2.16), (2.17), (2.19). The resulting determinant reads as

$$
D_{n}(M q)=\operatorname{det} \rho_{i j}(M q)
$$

with degeneracy $n^{2}$, where $\rho_{i j}$ is a $4 \times 4$ matrix with entries

$$
\rho_{11}=I_{n}(M q)-M q I_{n}^{\prime}(M q), \rho_{12}=M q I_{n}^{\prime}(M q)
$$




$$
\begin{gathered}
\rho_{13}=(2-n) I_{n-2}(M q)+M q I_{n-2}^{\prime}(M q), \rho_{14}=(2+n) I_{n+2}(M q)+M q I_{n+2}^{\prime}(M q), \\
\rho_{21}=-\left(n^{2}-1\right) I_{n}(M q), \rho_{22}=2 M q I_{n}^{\prime}(M q)+6 I_{n}(M q), \\
\rho_{23}=2(n+1) M q I_{n-2}^{\prime}(M q)-\left(n^{2}-6 n-7\right) I_{n-2}(M q), \\
\rho_{24}=-2(n-1) M q I_{n+2}^{\prime}(M q)-\left(n^{2}+6 n-7\right) I_{n+2}(M q), \\
\rho_{31}=0, \rho_{32}=-I_{n}(M q), \\
\rho_{33}=\frac{(n+1)}{(n-2)} I_{n-2}(M q), \rho_{34}=\frac{(n-1)}{(n+2)} I_{n+2}(M q), \\
\rho_{41}=3 I_{n}(M q), \rho_{42}=-2 I_{n}(M q), \rho_{43}=-I_{n-2}(M q), \rho_{44}=-I_{n+2}(M q) .
\end{gathered}
$$

Transverse-traceless tensor modes $k_{n}(\tau)$ yield, by virtue of Eqs. (2.10) and (2.19),

$$
D_{n}(M q)=I_{n}(M q), \forall n \geq 3
$$

with degeneracy $2\left(n^{2}-4\right)$.

As far as ghost modes are concerned, the decoupled mode $l_{1}(\tau)=\frac{1}{\tau} I_{2}(M \tau)$ vanishes at the three-sphere boundary and hence yields

$$
D(M q)=I_{2}(M q)
$$

with degeneracy 1 , while scalar and vector ghost modes lead to

$$
D_{n}(M q)=I_{n+1}(M q), \quad \forall n \geq 2
$$

and

$$
D_{n}(M q)=I_{n}(M q), \quad \forall n \geq 2,
$$

respectively, with degeneracy $n^{2}$ for Eq. (2.42) and $2\left(n^{2}-1\right)$ for Eq. (2.43). 
Our $D(M q)$ equations can be re-expressed in a very helpful way by using repeatedly the identities for modified Bessel functions and their derivatives in the Appendix. Hence we find, on setting $w \equiv M q$, that

$$
\frac{D(w)}{2}=I_{2}(w)
$$

for the decoupled vector mode in Eq. (2.20), while coupled vector modes in Eq. (2.21) yield

$$
\frac{(n+2)}{4 n} D_{n}(w)=I_{n}(w)\left(I_{n}^{\prime}(w)+\frac{2}{w} I_{n}(w)\right) .
$$

Moreover, the scalar modes $a_{1}, e_{1}$ ruled by Eq. (2.26) yield

$$
\frac{D(w)}{4 w}=I_{2}(w)\left(I_{2}^{\prime}(w)+\frac{4}{w} I_{2}(w)\right)
$$

and the scalar modes $a_{2}, b_{2}, e_{2}$ ruled by Eq. (2.30) lead to

$$
\frac{D(w)}{6 w^{2}}=I_{1}(w) I_{3}(w)\left(I_{3}^{\prime}(w)+\frac{5}{w} I_{3}(w)\right) .
$$

\section{MATRIX FOR COUPLED SCALAR MODES}

The hardest part of our analysis is the investigation of Eq. (2.31). For this purpose, we first exploit the formulae in Appendix B to find

$$
\begin{gathered}
\rho_{11}=I_{n}(w)-w I_{n}^{\prime}(w), \rho_{12}=w I_{n}^{\prime}(w), \rho_{13}=w I_{n}^{\prime}(w)+n I_{n}(w), \rho_{14}=w I_{n}^{\prime}(w)-n I_{n}(w), \quad(3.1) \\
\rho_{21}=-\left(n^{2}-1\right) I_{n}(w), \rho_{22}=2\left(w I_{n}^{\prime}(w)+3 I_{n}(w)\right), \\
\rho_{23}=(n+1)\left\{\left[3(n+1)+\frac{2 n(n-1)(n+3)}{w^{2}}\right] I_{n}(w)+2\left[w+\frac{(n-1)(n+3)}{w}\right] I_{n}^{\prime}(w)\right\}, \\
\rho_{24}=(n-1)\left\{\left[3(n-1)+\frac{2 n(n+1)(n-3)}{w^{2}}\right] I_{n}(w)-2\left[w+\frac{(n+1)(n-3)}{w}\right] I_{n}^{\prime}(w)\right\}, \\
\rho_{31}=0, \rho_{32}=-I_{n}(w),
\end{gathered}
$$




$$
\begin{gathered}
\rho_{33}=\frac{(n+1)}{(n-2)}\left[\left(1+\frac{2 n(n-1)}{w^{2}}\right) I_{n}(w)+\frac{2(n-1)}{w} I_{n}^{\prime}(w)\right], \\
\rho_{34}=\frac{(n-1)}{(n+2)}\left[\left(1+\frac{2 n(n+1)}{w^{2}}\right) I_{n}(w)-\frac{2(n+1)}{w} I_{n}^{\prime}(w)\right], \\
\rho_{41}=3 I_{n}(w), \rho_{42}=-2 I_{n}(w), \\
\rho_{43}=-\left(1+\frac{2 n(n-1)}{w^{2}}\right) I_{n}(w)-\frac{2(n-1)}{w} I_{n}^{\prime}(w), \\
\rho_{44}=-\left(1+\frac{2 n(n+1)}{w^{2}}\right) I_{n}(w)+\frac{2(n+1)}{w} I_{n}^{\prime}(w) .
\end{gathered}
$$

The resulting determinant, despite its cumbersome expression, can be studied by introducing the variable

$$
y \equiv \frac{I_{n}^{\prime}(w)}{I_{n}(w)}
$$

which leads to

$$
D_{n}(w)=\frac{48 n\left(1-n^{2}\right)}{\left(n^{2}-4\right)} I_{n}^{4}(w)\left(y-y_{1}\right)\left(y-y_{2}\right)\left(y-y_{3}\right)\left(y-y_{4}\right)
$$

where

$$
y_{1} \equiv-\frac{n}{w}, y_{2} \equiv \frac{n}{w}, y_{3} \equiv-\frac{n}{w}-\frac{w}{2}, y_{4} \equiv \frac{n}{w}-\frac{w}{2},
$$

and hence

$$
\begin{aligned}
\frac{\left(n^{2}-4\right)}{48 n\left(1-n^{2}\right)} D_{n}(w) & =\left(I_{n}^{\prime}(w)+\frac{n}{w} I_{n}(w)\right)\left(I_{n}^{\prime}(w)-\frac{n}{w} I_{n}(w)\right) \\
& \times\left(I_{n}^{\prime}(w)+\left(\frac{w}{2}+\frac{n}{w}\right) I_{n}(w)\right)\left(I_{n}^{\prime}(w)+\left(\frac{w}{2}-\frac{n}{w}\right) I_{n}(w)\right)
\end{aligned}
$$

\section{FIRST PAIR OF SCALAR-MODE $\zeta$-FUNCTIONS}

Equations (2.40)-(2.47) and (3.14) are sufficient to obtain an integral representation of the $\zeta$-function, the residues of which yield all heat-kernel coefficients. This topic is described in great detail in the existing literature (see, for example, Refs. [11] and [15]) and hence we limit ourselves to a very brief outline before presenting our results. 
Given the elliptic operator $P$ acting on physical fields defined on the $m$-dimensional Riemannian manifold $\mathcal{M}$, one can build the associated heat kernel $U(x, y ; t)$ and the corresponding integrated heat kernel (bundle indices are not written down explicitly, but the fibre trace tr takes them into account)

$$
\operatorname{Tr}_{L^{2}} e^{-t P}=\int_{\mathcal{M}} \operatorname{tr} U(x, x ; t) \sqrt{g} d^{m} x
$$

which has the asymptotic expansion, as $t \rightarrow 0^{+}$,

$$
\operatorname{Tr}_{L^{2}} e^{-t P} \sim(4 \pi t)^{-\frac{m}{2}} \sum_{k=0}^{\infty} A_{\frac{k}{2}} t^{\frac{k}{2}}
$$

The $A_{\frac{k}{2}}$ coefficients are said to describe the global asymptotics in that they are obtained by integration over $\mathcal{M}$ and its boundary $\mathcal{B}$ of local geometric invariants built from the Riemann curvature of $\mathcal{M}$, gauge curvature, extrinsic curvature of $\mathcal{B}$, potential terms in $P$ and in the boundary operator expressing the boundary conditions. On the other hand, since the $\zeta$ function of $P$ is related to the integrated heat kernel by an inverse Mellin transform $[1,3,11]$ :

$$
\zeta_{P}(s)=\operatorname{Tr}_{L^{2}} P^{-s}=\sum_{\lambda_{P}} \lambda_{P}^{-s}=\frac{1}{\Gamma(s)} \int_{0}^{\infty} t^{s-1}\left(\operatorname{Tr}_{L^{2}} e^{-t P}\right) d t
$$

the global heat-kernel coefficients in the asymptotic expansion (4.2) can be also obtained from the residues of $\zeta_{P}(s)[15]$.

Moreover, since the function occurring in the equation obeyed by the eigenvalues by virtue of the boundary conditions admits a canonical-product representation $[1,3]$, one can also express $\zeta_{P}(s)$ as a contour integral which is eventually rotated to the imaginary axis. The residues of the latter integral yield therefore the $A_{\frac{k}{2}}$ coefficients used in evaluating one-loop effective action and one-loop divergences.

In our problem the $P$ operator is the Laplacian on the Euclidean four-ball acting on metric perturbations. Equations (2.40)-(2.47) correspond to a familiar mixture of Dirichlet and Robin boundary conditions for which integral representation of the $\zeta$-function and heatkernel coefficients are immediately obtained. New features arise instead from Eq. (3.14), that gives rise, at first sight, to four different $\zeta$-functions. On studying the first line of Eq. (3.14), we can exploit the work in Ref. [16] and the uniform asymptotic expansion of Bessel functions and their first derivatives (see Appendix B) to say that the integral representation 
of the resulting $\zeta$-function reads as

$$
\zeta_{A}^{ \pm}(s) \equiv \frac{(\sin \pi s)}{\pi} \sum_{n=3}^{\infty} n^{-(2 s-2)} \int_{0}^{\infty} d z z^{-2 s} \frac{\partial}{\partial z} \log \left[z^{-\beta_{ \pm}(n)}\left(z n I_{n}^{\prime}(z n) \pm n I_{n}(z n)\right)\right] .
$$

With our notation, $\beta_{+}(n)=n, \beta_{-}(n)=n+2$, where these factors are fixed by the leading behaviour of the eigenvalue condition as $z \rightarrow 0$ [15]; the uniform asymptotic expansion of modified Bessel functions and their first derivatives (see Appendix B) can be used to find (hereafter $\tau=\tau(z) \equiv\left(1+z^{2}\right)^{-\frac{1}{2}}$ from Eq. (B8))

$$
z n I_{n}^{\prime}(z n) \pm n I_{n}(z n) \sim \frac{n}{\sqrt{2 \pi n}} \frac{e^{n \eta}}{\sqrt{\tau}}(1 \pm \tau)\left(1+\sum_{k=1}^{\infty} \frac{p_{k, \pm}(\tau)}{n^{k}}\right)
$$

where

$$
p_{k, \pm}(\tau) \equiv(1 \pm \tau)^{-1}\left(v_{k}(\tau) \pm \tau u_{k}(\tau)\right)
$$

for all $k \geq 1$, and

$$
\log \left(1+\sum_{k=1}^{\infty} \frac{p_{k, \pm}(\tau)}{n^{k}}\right) \sim \sum_{k=1}^{\infty} \frac{T_{k, \pm}(\tau)}{n^{k}}
$$

Thus, the $\zeta$-functions (4.4) obtain, from the first pair of round brackets in Eq. (4.5), the contributions (cf. Ref. [16])

$$
\begin{gathered}
A_{+}(s) \equiv \sum_{n=3}^{\infty} n^{-(2 s-2)} \frac{(\sin \pi s)}{\pi} \int_{0}^{\infty} d z z^{-2 s} \frac{\partial}{\partial z} \log \left(1+\left(1+z^{2}\right)^{-\frac{1}{2}}\right) \\
A_{-}(s) \equiv \sum_{n=3}^{\infty} n^{-(2 s-2)} \frac{(\sin \pi s)}{\pi} \int_{0}^{\infty} d z z^{-2 s} \frac{\partial}{\partial z} \log \left(\frac{1-\left(1+z^{2}\right)^{-\frac{1}{2}}}{z^{2}}\right),
\end{gathered}
$$

where $z^{2}$ in the denominator of the argument of the log arises, in Eq. (4.9), from the extra $z^{-2}$ in the prefactor $z^{-\beta_{-}(n)}$ in the definition (4.4). Moreover, the second pair of round brackets in Eq. (4.5) contributes $\sum_{j=1}^{\infty} A_{j, \pm}(s)$, having defined

$$
A_{j, \pm}(s) \equiv \sum_{n=3}^{\infty} n^{-(2 s+j-2)} \frac{(\sin \pi s)}{\pi} \int_{0}^{\infty} d z z^{-2 s} \frac{\partial}{\partial z} T_{j, \pm}(\tau(z)),
$$

where, from the formulae

$$
\begin{gathered}
T_{1, \pm}=p_{1, \pm} \\
T_{2, \pm}=p_{2, \pm}-\frac{1}{2} p_{1, \pm}^{2},
\end{gathered}
$$




$$
T_{3, \pm}=p_{3, \pm}-p_{1, \pm} p_{2, \pm}+\frac{1}{3} p_{1, \pm}^{3}
$$

we find

$$
\begin{gathered}
T_{1, \pm}=-\frac{3}{8} \tau \pm \frac{1}{2} \tau^{2}-\frac{5}{24} \tau^{3}, \\
T_{2, \pm}=-\frac{3}{16} \tau^{2} \pm \frac{3}{8} \tau^{3}+\frac{1}{8} \tau^{4} \mp \frac{5}{8} \tau^{5}+\frac{5}{16} \tau^{6}, \\
T_{3, \pm}=-\frac{21}{128} \tau^{3} \pm \frac{3}{8} \tau^{4}+\frac{509}{640} \tau^{5} \mp \frac{25}{12} \tau^{6}+\frac{21}{128} \tau^{7} \pm \frac{15}{8} \tau^{8}-\frac{1105}{1152} \tau^{9},
\end{gathered}
$$

and hence, in general,

$$
T_{j, \pm}(\tau)=\sum_{a=j}^{3 j} f_{a}^{(j, \pm)} \tau^{a}
$$

We therefore find, from the first line of Eq. (3.14), contributions to the generalized $\zeta$-function, from terms in round brackets in Eq. (4.5), equal to

$$
\delta \zeta_{A}^{ \pm}(s)=\omega_{0}(s) F_{0}^{ \pm}(s)+\sum_{j=1}^{\infty} \omega_{j}(s) F_{j}^{ \pm}(s)
$$

where, for all $\lambda=0, j$,

$$
\omega_{\lambda}(s) \equiv \sum_{n=3}^{\infty} n^{-(2 s+\lambda-2)}=\zeta_{H}(2 s+\lambda-2 ; 3)=\zeta_{R}(2 s+\lambda-2)-1-2^{-(2 s+\lambda-2)},
$$

while, from Eqs. (4.8)-(4.10),

$$
\begin{gathered}
F_{0}^{+}(s) \equiv \frac{(\sin \pi s)}{\pi} \int_{0}^{\infty} d z z^{-2 s} \frac{\partial}{\partial z} \log \left(1+\left(1+z^{2}\right)^{-\frac{1}{2}}\right), \\
F_{0}^{-}(s) \equiv-2 \frac{(\sin \pi s)}{\pi} \int_{0}^{\infty} d z \frac{z^{-(2 s-1)}}{\left(1+z^{2}\right)}-F_{0}^{+}(s)=-1-F_{0}^{+}(s), \\
F_{j}^{ \pm}(s) \equiv \frac{(\sin \pi s)}{\pi} \sum_{a=j}^{3 j} L^{ \pm}(s, a, 0) f_{a}^{(j, \pm)},
\end{gathered}
$$

having set (this general definition will prove useful later, and arises from a more general case, where $\tau^{a}$ is divided by the $b$-th power of $(1 \pm \tau)$ in Eq. (4.17))

$$
L^{ \pm}(s, a, b) \equiv \int_{0}^{1} \tau^{2 s+a}(1-\tau)^{-s}(1+\tau)^{-s}\left( \pm b(1 \pm \tau)^{-b-1}-a \tau^{-1}(1 \pm \tau)^{-b}\right) d \tau .
$$


Moreover, on considering

$$
L_{0}^{+}(s) \equiv \frac{\pi}{\sin \pi s} F_{0}^{+}(s),
$$

and changing variable from $z$ to $\tau$ therein, all $L$-type integrals above can be obtained from

$$
Q(\alpha, \beta, \gamma) \equiv \int_{0}^{1} \tau^{\alpha}(1-\tau)^{\beta}(1+\tau)^{\gamma} d \tau
$$

In particular, we will need

$$
\begin{gathered}
L_{0}^{+}(s)=-Q(2 s,-s,-s-1), \\
L^{+}(s, a, b)=b Q(2 s+a,-s,-s-b-1)-a Q(2 s+a-1,-s,-s-b),
\end{gathered}
$$

where, from the integral representation of the hypergeometric function, one has [17]

$$
Q(\alpha, \beta, \gamma)=\frac{\Gamma(\alpha+1) \Gamma(\beta+1)}{\Gamma(\alpha+\beta+2)} F(-\gamma, \alpha+1 ; \alpha+\beta+2 ;-1) .
$$

For example, explicitly,

$$
L_{0}^{+}(s)=-\frac{\Gamma(2 s+1) \Gamma(1-s)}{\Gamma(s+2)} F(s+1,2 s+1 ; s+2 ;-1) .
$$

Now we exploit Eqs. (4.4), (4.5) and (4.18) to write

$$
\zeta_{A}^{+}(s)=\delta \zeta_{A}^{+}(s)+\frac{(\sin \pi s)}{\pi} \sum_{n=3}^{\infty} n^{-(2 s-2)} \int_{0}^{\infty} d z\left[\frac{z^{-(2 s-1)}}{2\left(1+z^{2}\right)}+n z^{-(2 s+1)}\left(\sqrt{1+z^{2}}-1\right)\right] .
$$

Hence we find

$$
\zeta_{A}^{+}(0)=\lim _{s \rightarrow 0}\left[\omega_{0}(s) F_{0}^{+}(s)+\sum_{j=1}^{\infty} \omega_{j}(s) F_{j}^{+}(s)+\left(\zeta_{A}^{+}(s)-\delta \zeta_{A}^{+}(s)\right)\right] .
$$

The first limit in Eq. (4.31) is immediately obtained by noting that

$$
\lim _{s \rightarrow 0} L_{0}^{+}(s)=-\log (2)
$$

and hence

$$
\lim _{s \rightarrow 0} \omega_{0}(s) F_{0}^{+}(s)=\lim _{s \rightarrow 0}\left[\zeta_{H}(2 s-2 ; 3) \frac{(\sin \pi s)}{\pi} L_{0}^{+}(s)\right]=0 .
$$

To evaluate the second limit in Eq. (4.31), we use

$$
\lim _{s \rightarrow 0} L^{+}(s, a, 0)=-1,
$$


and bear in mind that $\omega_{j}(s)$ is a meromorphic function with first-order pole, as $s \rightarrow 0$, only at $j=3$ by virtue of the limit

$$
\lim _{y \rightarrow 1}\left[\zeta_{R}(y)-\frac{1}{(y-1)}\right]=\gamma .
$$

Hence we find (see coefficients in Eq. (4.16))

$$
\begin{aligned}
\lim _{s \rightarrow 0} \sum_{j=1}^{\infty} \omega_{j}(s) F_{j}^{+}(s) & =\lim _{s \rightarrow 0} \frac{(\sin \pi s)}{\pi} \sum_{j=1}^{\infty} \omega_{j}(s)\left[\sum_{a=j}^{3 j} L^{+}(s, a, 0) f_{a}^{(j,+)}\right] \\
& =-\frac{1}{2} \sum_{a=3}^{9} f_{a}^{(3,+)}=-\frac{1}{720}
\end{aligned}
$$

while, from Eqs. (4.30) and (4.28),

$$
\begin{aligned}
\lim _{s \rightarrow 0}\left(\zeta_{A}^{+}(s)-\delta \zeta_{A}^{+}(s)\right) & =\lim _{s \rightarrow 0}\left(\frac{1}{4} \zeta_{H}(2 s-2 ; 3)+\frac{1}{4 \sqrt{\pi}} \frac{\Gamma\left(s-\frac{1}{2}\right)}{\Gamma(s+1)} \zeta_{H}(2 s-3 ; 3)\right) \\
& =-\frac{5}{4}+\frac{1079}{240} .
\end{aligned}
$$

From Eqs. (4.31)-(4.37) we therefore obtain

$$
\zeta_{A}^{+}(0)=-\frac{5}{4}+\frac{1079}{240}-\frac{1}{2} \sum_{a=3}^{9} f_{a}^{(3,+)}=\frac{146}{45} .
$$

An analogous procedure leads to

$$
\zeta_{A}^{-}(0)=-\frac{5}{4}+\frac{1079}{240}+5-\frac{1}{2} \sum_{a=3}^{9} f_{a}^{(3,-)}=\frac{757}{90}
$$

where the +5 contribution results from Eq. (4.21) when exploited in Eq. (4.18). These results have been double-checked by using also the powerful analytic technique in Ref. [14].

\section{FURTHER SPECTRAL ASYMPTOTICS: ELLIPTIC AND NON-ELLIPTIC PARTS}

As a next step, the second line of Eq. (3.14) suggests considering $\zeta$-functions having the integral representation (cf. Eq. (4.4))

$$
\begin{aligned}
\zeta_{B}^{ \pm}(s) \equiv & \frac{(\sin \pi s)}{\pi} \sum_{n=3}^{\infty} n^{-(2 s-2)} \\
& \int_{0}^{\infty} d z z^{-2 s} \frac{\partial}{\partial z} \log \left[z^{-\beta_{ \pm}(n)}\left(z n I_{n}^{\prime}(z n)+\left(\frac{z^{2} n^{2}}{2} \pm n\right) I_{n}(z n)\right)\right] .
\end{aligned}
$$


To begin, we exploit again the uniform asymptotic expansion of modified Bessel functions and their first derivatives to find (cf. Eq. (4.5))

$$
z n I_{n}^{\prime}(z n)+\left(\frac{z^{2} n^{2}}{2} \pm n\right) I_{n}(z n) \sim \frac{n^{2}}{2 \sqrt{2 \pi n}} \frac{e^{n \eta}}{\sqrt{\tau}}\left(\frac{1}{\tau}-\tau\right)\left(1+\sum_{k=1}^{\infty} \frac{r_{k, \pm}(\tau)}{n^{k}}\right)
$$

where we have (bearing in mind that $u_{0}=v_{0}=1$ )

$$
r_{k, \pm}(\tau) \equiv u_{k}(\tau)+\frac{2 \tau}{\left(1-\tau^{2}\right)}\left(\left(v_{k-1}(\tau) \pm \tau u_{k-1}(\tau)\right)\right.
$$

for all $k \geq 1$. Hereafter we set

$$
\Omega \equiv \sum_{k=1}^{\infty} \frac{r_{k, \pm}(\tau(z))}{n^{k}}
$$

and rely upon the formula

$$
\log (1+\Omega) \sim \sum_{k=1}^{\infty}(-1)^{k+1} \frac{\Omega^{k}}{k}
$$

to evaluate the uniform asymptotic expansion (cf. Eq. (4.7))

$$
\log \left(1+\sum_{k=1}^{\infty} \frac{r_{k, \pm}(\tau(z))}{n^{k}}\right) \sim \sum_{k=1}^{\infty} \frac{R_{k, \pm}(\tau(z))}{n^{k}}
$$

The formulae yielding $R_{k, \pm}$ from $r_{k, \pm}$ are exactly as in Eqs. (4.11)-(4.13), with $T$ replaced by $R$ and $p$ replaced by $r$ (see, however, comments below Eq. (5.10)). Hence we find, bearing in mind Eq. (5.3),

$$
\begin{gathered}
R_{1, \pm}=(1 \mp \tau)^{-1}\left(\frac{17}{8} \tau \mp \frac{1}{8} \tau^{2}-\frac{5}{24} \tau^{3} \pm \frac{5}{24} \tau^{4}\right) \\
R_{2, \pm}=(1 \mp \tau)^{-2}\left(-\frac{47}{16} \tau^{2} \pm \frac{15}{8} \tau^{3}-\frac{21}{16} \tau^{4} \pm \frac{3}{4} \tau^{5}-\frac{1}{16} \tau^{6} \mp \frac{5}{8} \tau^{7}+\frac{5}{16} \tau^{8}\right) \\
R_{3, \pm}=(1 \mp \tau)^{-3}\left(\frac{1721}{384} \tau^{3} \mp \frac{441}{128} \tau^{4}+\frac{597}{320} \tau^{5} \mp \frac{1033}{960} \tau^{6}+\frac{239}{80} \tau^{7}\right. \\
\left.\mp \frac{28}{5} \tau^{8}+\frac{2431}{576} \tau^{9} \pm \frac{221}{192} \tau^{10}-\frac{1105}{384} \tau^{11} \pm \frac{1105}{1152} \tau^{12}\right)
\end{gathered}
$$

and therefore

$$
R_{j, \pm}(\tau(z))=(1 \mp \tau)^{-j} \sum_{a=j}^{4 j} C_{a}^{(j, \pm)} \tau^{a}
$$

where, unlike what happens for the $T_{j, \pm}$ polynomials, the exponent of $(1 \mp \tau)$ never vanishes. Note that, at $\tau=1$ (i.e. $z=0$ ), our $r_{k,+}(\tau)$ and $R_{k,+}(\tau)$ are singular. Such a behaviour 
is not seen for any of the strongly elliptic boundary-value problems (see third item in Ref. 11]). This technical difficulty motivates our efforts below and is interpreted by us as a clear indication of the lack of strong ellipticity proved, on general ground, in Ref. [6].

The $\zeta_{B}^{-}(s)$ function is more easily dealt with. It indeed receives contributions from terms in round brackets in Eq. (5.2) equal to (cf. Eq. (4.9) and bear in mind that $\beta_{-}-\beta_{+}=2$ in Eq. (5.1))

$$
\begin{aligned}
B_{-}(s) & \equiv \sum_{n=3}^{\infty} n^{-(2 s-2)} \frac{(\sin \pi s)}{\pi} \int_{0}^{\infty} d z z^{-2 s} \frac{\partial}{\partial z} \log \left(\frac{\frac{1}{\tau(z)}-\tau(z)}{z^{2}}\right) \\
& =\omega_{0}(s) \frac{(\sin \pi s)}{\pi} \int_{0}^{\infty} d z z^{-2 s} \frac{\partial}{\partial z} \log \frac{1}{\sqrt{1+z^{2}}}=-\frac{1}{2} \omega_{0}(s)
\end{aligned}
$$

and $\sum_{j=1}^{\infty} B_{j,-}(s)$, having defined (cf. Eq. (4.10))

$$
B_{j,-}(s) \equiv \omega_{j}(s) \frac{(\sin \pi s)}{\pi} \int_{0}^{\infty} d z z^{-2 s} \frac{\partial}{\partial z} R_{j,-}(\tau(z))=\omega_{j}(s) \frac{(\sin \pi s)}{\pi} \sum_{a=j}^{4 j} L^{+}(s, a, j) C_{a}^{(j,-)} .
$$

On using the same method as in Sec. IV, the formulae (5.1)-(5.12) lead to (we find $L^{+}(0, a, 3)=-\frac{1}{8}$, independent of $a$, below $)$

$$
\zeta_{B}^{-}(0)=-\frac{5}{4}+\frac{1079}{240}+\frac{5}{2}+\frac{1}{2} \sum_{a=3}^{12} L^{+}(0, a, 3) C_{a}^{(3,-)}=\frac{206}{45},
$$

a result which agrees with a derivation of $\zeta_{B}^{-}(0)$ relying upon the method of Ref. [14].

Although we have stressed after Eq. (5.10) the problems with the $\zeta_{B}^{+}(s)$ part, for the moment let us proceed formally in the same way as above. Thus we define, in analogy to Eq. (5.11),

$$
B_{+}(s) \equiv \omega_{0}(s) \frac{(\sin \pi s)}{\pi} \int_{0}^{\infty} d z z^{-2 s} \frac{\partial}{\partial z} \log \left(\frac{1}{\tau(z)}-\tau(z)\right),
$$

and, in analogy to Eq. (5.12),

$$
B_{j,+}(s) \equiv \omega_{j}(s) \frac{(\sin \pi s)}{\pi} \int_{0}^{\infty} d z z^{-2 s} \frac{\partial}{\partial z} R_{j,+}(\tau(z)) .
$$

In order to make the presentation as transparent as possible, we write out the derivatives of $R_{j,+}$. On changing integration variable from $z$ to $\tau$ we define

$$
C_{j}(\tau) \equiv \frac{\partial}{\partial \tau} R_{j,+}(\tau)
$$

and we find the following results:

$$
C_{1}(\tau)=(1-\tau)^{-2}\left(\frac{17}{8}-\frac{1}{4} \tau-\frac{1}{2} \tau^{2}+\frac{5}{4} \tau^{3}-\frac{5}{8} \tau^{4}\right)
$$




$$
\begin{gathered}
C_{2}(\tau)=(1-\tau)^{-3}\left(-\frac{47}{8} \tau+\frac{45}{8} \tau^{2}-\frac{57}{8} \tau^{3}+\frac{51}{8} \tau^{4}-\frac{21}{8} \tau^{5}-\frac{33}{8} \tau^{6}+\frac{45}{8} \tau^{7}-\frac{15}{8} \tau^{8}\right) \\
C_{3}(\tau)=(1-\tau)^{-4}\left(\frac{1721}{128} \tau^{2}-\frac{441}{32} \tau^{3}+\frac{1635}{128} \tau^{4}-\frac{163}{16} \tau^{5}+\frac{1545}{64} \tau^{6}-\frac{227}{4} \tau^{7}\right. \\
\left.+\frac{4223}{64} \tau^{8}-\frac{221}{16} \tau^{9}-\frac{5083}{128} \tau^{10}+\frac{1105}{32} \tau^{11}-\frac{1105}{128} \tau^{12}\right)
\end{gathered}
$$

so that the general expression of $C_{j}(\tau)$ reads as

$$
C_{j}(\tau)=\sum_{a=j-1}^{4 j} \frac{K_{a}^{(j)} \tau^{a}}{(1-\tau)^{j+1}}, \forall j=1, \ldots, \infty .
$$

These formulae engender a $\zeta_{B}^{+}(0)$ which can be defined, after change of variable from $z$ to $\tau$, by splitting the integral with respect to $\tau$, in the integral representation of $\zeta_{B}^{+}(s)$, according to the identity

$$
\int_{0}^{1} d \tau=\int_{0}^{\mu} d \tau+\int_{\mu}^{1} d \tau
$$

and taking the limit as $\mu \rightarrow 1$ after having evaluated the integral. More precisely, since the integral on the left-hand side is independent of $\mu$, we can choose $\mu$ small on the right-hand side so that, in the interval $[0, \mu]$ (and only there!), we can use the uniform asymptotic expansion of the integrand where the negative powers of $(1-\tau)$ are harmless. Moreover, independence of $\mu$ also implies that, after having evaluated the integrals on the right-hand side, we can take the $\mu \rightarrow 1$ limit. Within this framework, the limit as $\mu \rightarrow 1$ of the second integral on the right-hand side yields vanishing contribution to the asymptotic expansion of $\zeta_{B}^{+}(s)$.

With this caveat, on defining (cf. Eq. (4.25))

$$
Q_{\mu}(\alpha, \beta, \gamma) \equiv \int_{0}^{\mu} \tau^{\alpha}(1-\tau)^{\beta}(1+\tau)^{\gamma} d \tau
$$

we obtain the representations

$$
\begin{gathered}
B_{+}(s)=-\omega_{0}(s) \frac{(\sin \pi s)}{\pi}\left[-Q_{\mu}(2 s,-s-1,-s)+Q_{\mu}(2 s,-s,-s-1)-Q_{\mu}(2 s-1,-s,-s)\right], \\
B_{j,+}(s)=-\omega_{j}(s) \frac{(\sin \pi s)}{\pi} \sum_{a=j-1}^{4 j} K_{a}^{(j)} Q_{\mu}(2 s+a,-s-j-1,-s) .
\end{gathered}
$$


The relevant properties of $Q_{\mu}(\alpha, \beta, \gamma)$ can be obtained by observing that this function is nothing but a hypergeometric function of two variables [17], i.e.

$$
Q_{\mu}(\alpha, \beta, \gamma)=\frac{\mu^{\alpha+1}}{\alpha+1} F_{1}(\alpha+1,-\beta,-\gamma, \alpha+2 ; \mu,-\mu)
$$

In detail, a summary of results needed to consider the limiting behaviour of $\zeta_{B}^{+}(s)$ as $s \rightarrow 0$ is

$$
\begin{gathered}
\omega_{0}(s) \frac{(\sin \pi s)}{\pi} \sim-5 s+\mathrm{O}\left(s^{2}\right) \\
\omega_{j}(s) \frac{(\sin \pi s)}{\pi} \sim \frac{1}{2} \delta_{j, 3}+\tilde{b}_{j, 1} s+\mathrm{O}\left(s^{2}\right) \\
\lim _{\mu \rightarrow 1} Q_{\mu}(2 s,-s-1,-s) \sim-\frac{1}{s}+\mathrm{O}\left(s^{0}\right) \\
\lim _{\mu \rightarrow 1} Q_{\mu}(2 s,-s,-s-1) \sim \log (2)+\mathrm{O}(s) \\
\lim _{\mu \rightarrow 1} Q_{\mu}(2 s-1,-s,-s) \sim \frac{1}{2 s}+\mathrm{O}(s) \\
\lim _{\mu \rightarrow 1} Q_{\mu}(2 s+a,-s-j-1,-s)=\frac{\Gamma(-j-s) \Gamma(a+2 s+1)}{\Gamma(a-j+s+1)} F(a+2 s+1, s, a-j+s+1 ;-1) \\
\sim \frac{b_{j,-1}(a)}{s}+b_{j, 0}(a)+\mathrm{O}(s)
\end{gathered}
$$

where

$$
\begin{gathered}
\tilde{b}_{j, 1}=-1-2^{2-j}+\zeta_{R}(j-2)\left(1-\delta_{j, 3}\right)+\gamma \delta_{j, 3}, \\
b_{j,-1}(a)=\frac{(-1)^{j+1}}{j !} \frac{\Gamma(a+1)}{\Gamma(a-j+1)}\left(1-\delta_{a, j-1}\right),
\end{gathered}
$$

and we only strictly need $b_{3,0}(a)$ which, unlike the elliptic cases studied earlier, now depends explicitly on $a$. In our case, we find

$$
\begin{gathered}
b_{3,0}(2)=\frac{1}{24}, b_{3,0}(3)=\frac{2}{3}-\log (2), b_{3,0}(4)=\frac{67}{24}-4 \log (2), b_{3,0}(5)=\frac{95}{12}-10 \log (2), \\
b_{3,0}(6)=\frac{143}{8}-20 \log (2), b_{3,0}(7)=\frac{139}{4}-35 \log (2), b_{3,0}(8)=\frac{2433}{40}-56 \log (2), \\
b_{3,0}(9)=\frac{1971}{20}-84 \log (2), b_{3,0}(10)=\frac{8429}{56}-120 \log (2),
\end{gathered}
$$




$$
b_{3,0}(11)=\frac{12289}{56}-165 \log (2), b_{3,0}(12)=\frac{155315}{504}-220 \log (2) .
$$

Remarkably, the coefficient of $\frac{1}{s}$ in the small-s behaviour of the generalized $\zeta$-function $\zeta_{B}^{+}(s)$ is zero because it is equal to

$$
\sum_{a=2}^{12} b_{3,-1}(a) K_{a}^{(3)}=\frac{1}{6} \sum_{a=3}^{12} a(a-1)(a-2) K_{a}^{(3)},
$$

which vanishes by virtue of the rather peculiar general property

$$
\sum_{a=j}^{4 j} \frac{\Gamma(a+1)}{\Gamma(a-j+1)} K_{a}^{(j)}=\sum_{a=j}^{4 j} \prod_{l=0}^{j-1}(a-l) K_{a}^{(j)}=0, \forall j=1, \ldots, \infty,
$$

and hence we find eventually

$$
\begin{aligned}
\zeta_{B}^{+}(0) & =-\frac{5}{4}+\frac{1079}{240}+\frac{5}{2}-\frac{1}{2} \sum_{a=2}^{12} b_{3,0}(a) K_{a}^{(3)}-\sum_{j=1}^{\infty} \tilde{b}_{j, 1} \sum_{a=j-1}^{4 j} b_{j,-1}(a) K_{a}^{(j)} \\
& =\frac{5}{4}+\frac{1079}{240}+\frac{599}{720}=\frac{296}{45}
\end{aligned}
$$

because the infinite sum on the first line of Eq. (5.34) vanishes by virtue of Eqs. (5.32) and (5.33), and exact cancellation of $\log (2)$ terms is found to occur.

To cross-check our analysis we remark that, on applying the technique of Ref. [14], one finds

$$
\zeta_{B}^{+}(0)=-\frac{15}{4}+\frac{1079}{240}-\frac{1}{720}=\frac{67}{90},
$$

where $-\frac{1}{720}$ results from working in the $n \rightarrow \infty$ and $w \rightarrow 0$ limit in

$$
\left(I_{n}^{\prime}(w)+\left(\frac{w}{2}+\frac{n}{w}\right) I_{n}(w)\right)
$$

on the second line of Eq. (3.14); such a term then reduces to $\left(I_{n}^{\prime}(w)+\frac{n}{w} I_{n}(w)\right)$. A possible interpretation of the discrepancy between (5.34) and (5.35) is that, when strong ellipticity is violated, prescriptions for defining a $\zeta(0)$ value exist but are inequivalent.

Remaining contributions to $\zeta(0)$, being obtained from strongly elliptic sectors of the boundary-value problem, are instead found to agree with the results in Ref. 7], i.e.

$$
\begin{gathered}
\zeta(0)[\text { transverse traceless modes }]=-\frac{278}{45}, \\
\zeta(0)[\text { coupled vector modes }]=\frac{494}{45},
\end{gathered}
$$




$$
\begin{gathered}
\zeta(0)[\text { decoupled vector mode }]=-\frac{15}{2}, \\
\zeta(0)\left[\text { scalar modes }\left(a_{1}, e_{1} ; a_{2}, b_{2}, e_{2}\right)\right]=-17, \\
\zeta(0)[\text { scalar ghost modes }]=-\frac{149}{45}, \\
\zeta(0)[\text { vector ghost modes }]=\frac{77}{90}, \\
\zeta(0)[\text { decoupled ghost mode }]=\frac{5}{2} .
\end{gathered}
$$

\section{CONCLUDING REMARKS}

We have obtained the analytically continued eigenvalue conditions for metric perturbations on the Euclidean four-ball, in the presence of boundary conditions completely invariant under infinitesimal diffeomorphisms in the de Donder gauge and with $\alpha$ parameter set to 1 in Eq. (1.10). Second, this has made it possible to prove, for the first time in the literature, that only one sector of the scalar-mode determinant is responsible for lack of strong ellipticity of the boundary-value problem (see second line of Eq. (3.14) and the analysis in Secs. IV and V). The first novelty with respect to the work in Ref. [6] is a better understanding of the elliptic and non-elliptic sectors of spectral asymptotics for Euclidean quantum gravity. Moreover, as far as we know, the detailed spectral asymptotics for $\zeta$-functions of Secs. IV and $\mathrm{V}$ was missing in the literature. We have also shown that one can indeed obtain a regular $\zeta$-function asymptotics at small $s$ in the non-elliptic case by virtue of the remarkable identity (5.33), here obtained for the first time. Our prescription for the $\zeta(0)$ value differs from the result first obtained in Ref. 7], where, however, neither the strong ellipticity issue 6] nor the non-standard spectral asymptotics of our Sec. V had been considered.

From the point of view of general formalism of Euclidean quantum gravity, three alternative pictures seem therefore to emerge:

(i) The remarkable factorization of eigenvalue conditions, with resulting isolation of elliptic part of spectral asymptotics (transverse-traceless, vector and ghost modes, all modes in 
finite-dimensional sub-spaces and three of the four equations for scalar modes), suggests trying to re-assess functional integrals on manifolds with boundary, with the hope of being able to obtain unique results from the non-elliptic contribution. If this cannot be achieved, the two alternatives below should be considered again.

(ii) Luckock boundary conditions [18], which engender BRST-invariant amplitudes but are

not diffeomorphism invariant [3]. They have already been applied by Moss and Poletti [19], [20].

(iii) Non-local boundary conditions that lead to surface states in quantum cosmology and pseudo-differential operators on metric and ghost modes [21]. Surface states are particularly interesting since they describe a transition from quantum to classical regime in cosmology entirely ruled by the strong ellipticity requirement, while pseudo-differential operators are a source of technical complications.

There is therefore encouraging evidence in favour of Euclidean quantum gravity being able to drive further developments in quantum field theory, quantum cosmology and spectral asymptotics (see early mathematical papers in Refs. [22], 23]) in the years to come.

\section{APPENDIX A: STRONG ELLIPTICITY}

For an operator of Laplace type, the boundary-value problem is strongly elliptic with respect to the cone $\mathbf{C}-\mathbf{R}_{+}$if, for any cotangent vector $u$ on the boundary $\mathcal{B}$, for any $\lambda \in \mathbf{C}-\mathbf{R}_{+}$, for any pair $(u, \lambda) \neq(0,0)$, there exists a unique solution $\varphi$ of the differential equation $(r$ being the geodesic distance to the boundary $\mathcal{B})$

$$
\left[-\frac{\partial^{2}}{\partial r^{2}}+u_{k} u^{k}-\lambda\right] \varphi(r)=0,
$$

subject to the asymptotic condition

$$
\lim _{r \rightarrow \infty} \varphi(r)=0
$$

and to the boundary conditions (here $\varphi(r)=\chi e^{-\sigma r}$ with $\sigma \equiv \sqrt{u_{k} u^{k}-\lambda}$ )

$$
\pi \varphi(r=0)=\psi_{0}, i T \varphi(r=0)+(I-\pi) \varphi^{\prime}(r=0)=\psi_{1}
$$

where $\pi$ is the same projector as in Eq. (1.1), iT is the leading symbol of that part of the boundary operator which involves tangential derivatives, while $\psi_{0}$ and $\psi_{1}$ are arbitrary 
boundary data. Eventually, all this is equivalent to proving positivity of the matrix $I \sqrt{u_{k} u^{k}}-$ $i T$ [6].

\section{APPENDIX B: BESSEL FUNCTIONS}

In Sec. II we exploit the following identities obeyed by modified Bessel functions of first kind:

$$
\begin{gathered}
I_{n+1}(w)=I_{n}^{\prime}(w)-\frac{n}{w} I_{n}(w), \\
I_{n-1}(w)=I_{n}^{\prime}(w)+\frac{n}{w} I_{n}(w), \\
I_{n+2}(w)=\left(1+\frac{2 n(n+1)}{w^{2}}\right) I_{n}(w)-\frac{2(n+1)}{w} I_{n}^{\prime}(w), \\
I_{n-2}(w)=\left(1+\frac{2 n(n-1)}{w^{2}}\right) I_{n}(w)+\frac{2(n-1)}{w} I_{n}^{\prime}(w), \\
I_{n+2}^{\prime}(w)=-\frac{2(n+1)}{w}\left(1+\frac{n(n+2)}{w^{2}}\right) I_{n}(w)+\left(1+\frac{2(n+1)(n+2)}{w^{2}}\right) I_{n}^{\prime}(w), \\
I_{n-2}^{\prime}(w)=\frac{2(n-1)}{w}\left(1+\frac{n(n-2)}{w^{2}}\right) I_{n}(w)+\left(1+\frac{2(n-1)(n-2)}{w^{2}}\right) I_{n}^{\prime}(w) .
\end{gathered}
$$

In Secs. IV and V we use the uniform asymptotic expansion of modified Bessel functions $I_{\nu}$ first found by Olver [24]:

$$
I_{\nu}(z \nu) \sim \frac{e^{\nu \eta}}{\sqrt{2 \pi \nu}\left(1+z^{2}\right)^{\frac{1}{4}}}\left(1+\sum_{k=1}^{\infty} \frac{u_{k}(\tau)}{\nu^{k}}\right),
$$

where

$$
\tau \equiv\left(1+z^{2}\right)^{-\frac{1}{2}}, \eta \equiv\left(1+z^{2}\right)^{\frac{1}{2}}+\log \left(\frac{z}{1+\sqrt{1+z^{2}}}\right) .
$$

This holds for $\nu \rightarrow \infty$ at fixed $z$. The polynomials $u_{k}(\tau)$ can be found from the recurrence relation [15]

$$
u_{k+1}(\tau)=\frac{1}{2} \tau^{2}\left(1-\tau^{2}\right) u_{k}^{\prime}(\tau)+\frac{1}{8} \int_{0}^{\tau} d \rho\left(1-5 \rho^{2}\right) u_{k}(\rho)
$$


starting with $u_{0}(\tau)=1$. Moreover, the first derivative of $I_{\nu}$ has the following uniform asymptotic expansion at large $\nu$ and fixed $z$ :

$$
I_{\nu}^{\prime}(z \nu) \sim \frac{e^{\nu \eta}}{\sqrt{2 \pi \nu}} \frac{\left(1+z^{2}\right)^{\frac{1}{4}}}{z}\left(1+\sum_{k=1}^{\infty} \frac{v_{k}(\tau)}{\nu^{k}}\right),
$$

with the $v_{k}$ polynomials determined from the $u_{k}$ according to 15$]$

$$
v_{k}(\tau)=u_{k}(\tau)+\tau\left(\tau^{2}-1\right)\left[\frac{1}{2} u_{k-1}(\tau)+\tau u_{k-1}^{\prime}(\tau)\right],
$$

starting with $v_{0}(\tau)=u_{0}(\tau)=1$.

\section{ACKNOWLEDGMENTS}

The present paper is dedicated to the loving memory of Mirella Russo and Bryce DeWitt. We are indebted to Ivan Avramidi for inspiration provided by previous collaboration with some of us and by continuous correspondence, and to Gerd Grubb for enlightening correspondence. K. Kirsten is grateful to the Baylor University Summer Sabbatical Program and to the INFN for financial support. The work of G. Esposito and K. Kirsten has been partially supported also by PRIN 2002 SINTESI.

[1] G. Esposito, Quantum Gravity, Quantum Cosmology and Lorentzian Geometries, Lecture Notes in Physics Vol. m12, LNPHA,M12,1 (Springer, Berlin, 1994).

[2] K. Milton, Quantum Field Theory Under the Influence of External Conditions (Rinton, Princeton, 2004).

[3] G. Esposito, A.Yu. Kamenshchik, and G. Pollifrone, Euclidean Quantum Gravity on Manifolds with Boundary, Fundamental Theories of Physics Vol. 85, FTPHD,85,1 (Kluwer, Dordrecht, 1997).

[4] A.O. Barvinsky, Phys. Lett. B 195, 344 (1987).

[5] I.G. Moss and P. Silva, Phys. Rev. D 55, 1072 (1997).

[6] I.G. Avramidi and G. Esposito, Commun. Math. Phys. 200, 495 (1999); Class. Quantum Grav. 15, 1141 (1998).

[7] G. Esposito, A.Yu. Kamenshchik, I.V. Mishakov, and G. Pollifrone, Phys. Rev. D 52, 3457 (1995). 
[8] I.G. Avramidi, G. Esposito, and A.Yu. Kamenshchik, Class. Quantum Grav. 13, 2361 (1996).

[9] B.S. DeWitt, The Global Approach to Quantum Field Theory (Oxford University Press, Oxford, 2003).

[10] K. Schleich, Phys. Rev. D 32, 1889 (1985).

[11] P.B. Gilkey, Invariance Theory, The Heat Equation and The Atiyah-Singer Index Theorem (CRC Press, Boca Raton, 1995); G. Esposito, Dirac Operators and Spectral Geometry, hep-th/9704016 (Cambridge University Press, Cambridge, 1998); K. Kirsten, Spectral Functions in Mathematics and Physics (CRC Press, Boca Raton, 2001).

[12] E.M. Lifshitz and I.M. Khalatnikov, Adv. Phys. 12, 185 (1963).

[13] G. Esposito, A.Yu. Kamenshchik, I.V. Mishakov, and G. Pollifrone, Phys. Rev. D 50, 6329 (1994).

[14] A.O. Barvinsky, A.Yu. Kamenshchik, and I.P. Karmazin, Ann. Phys. (N.Y.) 219, 201 (1992).

[15] M. Bordag, E. Elizalde and K. Kirsten, J. Math. Phys. 37, 895 (1996).

[16] J.S. Dowker and K. Kirsten, Class. Quantum Grav. 14, L169 (1997).

[17] I.S. Gradshteyn and I.M. Ryzhik, Table of Integrals, Series and Products (Academic Press, New York, 1965).

[18] H.C. Luckock, J. Math. Phys. 32, 1755 (1991).

[19] I.G. Moss and S. Poletti, Nucl. Phys. B 341, 155 (1990).

[20] I.G. Moss and S. Poletti, Phys. Lett. B 333, 326 (1994).

[21] V.N. Marachevsky and D.V. Vassilevich, Class. Quantum Grav. 13, 645 (1996); G. Esposito, Class. Quantum Grav. 16, 1113 (1999); Class. Quantum Grav. 16, 3999 (1999); Int. J. Mod. Phys. A 15, 4539 (2000).

[22] G. Grubb, Ann. Sc. N. Sup. Pisa, Ser. IV, 1, 1 (1974).

[23] P.B. Gilkey and L. Smith, J. Diff. Geom. 18, 393 (1983).

[24] F.W.J. Olver, Phil. Trans. R. Soc. Lond. A 247, 328 (1954). 